\title{
Nonlinear control of transport in drift wave turbulence
}

\author{
C. Brandt ${ }^{1}$, O. Grulke ${ }^{1,2, *}$, and T. Klinger ${ }^{1,2}$ \\ ${ }^{1}$ MPI for Plasma Physics, EURATOM Association, D-17491 Greifswald, Germany and \\ ${ }^{2}$ Ernst-Moritz-Arndt University, D-17489 Greifswald, Germany
}

\begin{abstract}
The control of the dynamics of drift waves is investigated experimentally in a linear laboratory plasma device. The control parameter is a mode selective drive of currents parallel to the magnetic field using an arrangement of eight azimuthally arranged electrodes and eight saddle coils, respectively. The driven currents resemble the intrinsic drift mode currents. Single coherent drift modes are synchronized in frequency by both control setups only if the control signal has the same mode structure and direction of phase velocity as the drift mode. The frequency range of synchronization depends nonlinearly on the amplitude of the driven current. In drift wave turbulence the turbulent fluctuations are synchronized to a single coherent drift mode to the expense of the energy of the broadband turbulent fluctuations. As a result the phase shift of density and potential fluctuations decreases considerably, thereby reducing the cross-field transport.
\end{abstract}

PACS:52.25.Fi, 52.25.Xz, 52.30.Ex, 52.35.Kt, 52.35.Ra

JNM keywords: Diffusion, Plasma Properties

PSI-17 keywords: Cross-Field Transport, Turbulence

* Corresponding author address:Wendelsteinstr. 1, Greifswald, Germany

* Corresponding author E-mail: grulke@ipp.mpg.de

Presenting author: O. Grulke

Presenting author E-mail: grulke@ipp.mpg.de 


\section{INTRODUCTION}

The fluctuation induced transport in the edge and scrape-off layer (SOL) of fusion devices is one of the key issues for next step fusion experiments since it represents a major challenge in the context of, e.g., confinement properties, plasma-wall interaction, impurity control, and the divertor concept. Although the mechanism of fluctuation-induced $E \times B$ transport does not change across the magnetic separatrix, the underlying instability mechanisms are believed to change significantly. In the SOL the open field line geometry leads to curvature-driven interchange mode dominated fluctuations, which are characterized by a large phase shift between plasma pressure and potential fluctuations and form essentially flute-like perturbations with $k_{\|}=0$ [1]. The dynamics changes considerably in the closed field line region of the plasma edge, which represents the interface region between the plasma core and the SOL. Here drift wave dynamics dominates the evolution of fluctuations. The destabilization of drift waves is intrinsically tight to the development of fluctuating parallel currents, leading to three-dimensional dynamics with variation along the magnetic field $k_{\|} \neq 0$ [2]. It is an appealing perspective to gain some control over the cross-field edge transport by influencing the drift wave dynamics itself. The successful application of several control schemes including chaos and feedback control in plasma dynamics have been reported [3-5]. In the present paper experimental studies of the mode-selective nonlinear control of drift wave turbulence are presented. The investigations are done in a linear laboratory experiment. The control scheme follows an open-loop concept, in which a spatiotemporal control signal is used [6].

\section{EXPERIMENT AND SETUP OF CONTROL SYSTEM}

The investigations are done in the linear, homogeneously magnetized helicon device VINETA. Argon plasma is produced via helicon wave heating with a rf-frequency of $f_{r f}=13.56 \mathrm{MHz}$ at a power of $P_{r f} \leq 5 \mathrm{~kW}$ and a magnetic field of $B=100 \mathrm{mT}$. The efficient helicon heating provides a high plasma density $n \leq 1 \cdot 10^{19} \mathrm{~m}^{-3}$ at a low electron temperature of $T_{e}=3 \mathrm{eV}$. In a series of experimental campaigns we have demonstrated that drift waves can be destabilized and can be driven from single linear coherent modes into weakly developed drift wave turbulence [7, 8]. The drift wave modes show all characteristics of linear modes: The associated plasma density fluctuations are localized in the radial density gradient region, the azimuthal propagation is in electron diamagnetic drift direction and the spatial mode structure is in very good agreement with linear dispersion calculations. Most important in the present context is a small phase shift $\varphi$ between density and plasma potential fluctuations, which are measured to be in the range $\varphi=\pi / 5-\pi / 10$. Consequently, the radial fluctuation-induced transport is small. The turbulent fluctuations are characterized by the formation of spatiotemporal fluctuation structures in plasma density and potential. In addition to the convection with the background azimuthal $E \times B$ plasma flow the turbulent struc- 
tures show a relatively strong radial component of propagation $v_{r} \approx 5 \% c_{s}$ [9], where $c_{s}$ is the local ion sound speed and thus resemble very much the propagation features of turbulent structures in the edge and SOL of tokamaks. In contrast to the linear modes the phase shift between plasma density and potential fluctuations is much larger $\varphi \approx \pi / 2$. The resulting cross-field transport is of burst-like nature and displays strong intermittency in the far plasma edge. To influence the drift wave fluctuations dynamically two control devices are used: The first system consists of an array of 8 electrodes, which surround the plasma column and are radially placed in the plasma edge outside the maximum radial density gradient region, comparable to the setup used by Schröder et al. [7]. The electrodes are localized along the magnetic and are connected to an 8 channel signal generator, which powers the electrodes with sinusoidal voltages of controllable phase shift. Depending on the chosen phase delay an azimuthally propagating wave pattern can be produced with mode numbers ranging from $m=1-3$. As a result a mode selective azimuthal pattern of parallel current can be driven by the electrodes. In Fig. 1 measurements of the parallel currents driven by the electrodes for a $m=2$ mode pattern and the intrinsic parallel drift wave currents of a coherent $m=2$ mode are compared for a single time instant. The azimuthal current pattern of the coherent drift mode shows the $m=2$ structure. The maximum current amplitudes reach $\left|j_{\|, d w}\right| \leq 100 \mathrm{~mA} / \mathrm{cm}^{2}$. The pattern shows an azimuthally bent structure, which resembles the pattern of density fluctuations of the mode [7]. The parallel currents driven by the electrodes has a also a $m=2$ mode pattern. Although the spatial position of the current pattern is located at larger radii an overlap of the positions of the drift mode and electrode driven currents are observed. The maximum current driven by the electrodes can reach amplitudes similar or even larger than the intrinsic drift mode currents and are limited only by the electron saturation current $\left|j_{\|, e l}\right| \leq\left|j_{e, s a t}\right|$ for a bias voltage amplitude of $V_{\text {bias }} \leq 20 \mathrm{~V}$. A similar result is found for the inductive parallel current drive using the azimuthal arrangement of control coils. However, due to power limitations of the control coil drive of $P_{\text {coil }} \leq 500 \mathrm{~W}$ the maximum parallel current amplitude driven by the control coils is significantly smaller than the intrinsic drift wave currents $\left|j_{\|, \text {coil }}\right| \leq 50 \mathrm{~mA} / \mathrm{cm}^{2}$.

\section{SYNCHRONIZATION OF LINEAR DRIFT MODES AND CONTROL OF DRIFT WAVE TURBULENCE}

The influence of parallel current driven by the control electrodes and the control coil setup, respectively, on the drift wave dynamics is demonstrated on single coherent modes. The results for a frequency scan of the control wave pattern across the drift mode frequency is shown in Fig. 2. The $m=2$ drift mode has a fixed frequency of $f=3 \mathrm{kHz}$. The frequency of the $m=2$ electrode control pattern is consecutively scanned in frequency in the range $f=2-5 \mathrm{kHz}$. The azimuthal propagation of the control pattern is in the same direction as the drift mode. The mode and control signals are coexisting for large values of the frequency mismatch. When the control signal 
frequency approaches the drift mode frequency the drift mode is synchronized by the control signal and its frequency follows strictly the control signal frequency for a certain range of frequency mismatch, until the drift mode locks out of the control signal. The range of mismatch, in which synchronization is found, is asymmetric to the mode frequency. A similar result is obtained for the control coil signal for a $m=2$ mode pattern. The mode frequency in this case is $f=7 \mathrm{kHz}$. The frequency range is again found to be strongly asymmetric to the drift mode frequency. Synchronization in both cases is only achieved if the control signal pattern is propagating azimuthally in the direction of the drift mode. For counter propagation no influence on the drift mode is observed. The range of synchronization is not fixed in frequency space but strongly depends on the amplitude of the control signal. In Fig. 2 the dependence of the synchronization range on the control signal amplitude for both control setups is shown. The asymmetry of the frequency range persists for all amplitudes. A broadening of the synchronization range is observed if the control amplitudes are increased. In the case of the control electrodes a linear increase of synchronization range with amplitude of the control signal is observed for amplitudes $\left|j_{\|, e l}\right| \leq 50 \mathrm{~mA} / \mathrm{cm}^{2}$. For larger amplitudes the synchronization range increases nonlinearly. A similar behavior is observed for the control coil setup. However, due to the technical limitations of the current amplitude only the linear increase is observed.

Due to the achievable higher amplitudes of the electrode driven currents, this setup has been used to control the drift wave turbulence fluctuations. The fluctuation characteristics of the turbulent state and the controlled state are compared in Fig. 3. In the turbulent state the power density spectrum is characterized by incoherent fluctuations without any mode signature and a power law decrease of the spectral power in the range $f=10-50 \mathrm{kHz}$. The driven control current pattern is chosen as the observed $m=2$ mode at a frequency of $f=7 k H z$ (c.f. Fig. 2). The resulting fluctuations show a $m=2$ mode structure, which is highly coherent as observed in the power spectrum at $f=7 \mathrm{kHz}$ and its higher harmonics as well as its subharmonic and the exposed peak in the wavenumberfrequency spectrum. The energy of the incoherent turbulent fluctuations decreases in favor of the $m=2$ mode, which fluctuation amplitude is slightly larger than the turbulent fluctuations. However, the phase shift between density and potential fluctuations decreases considerable as shown in Fig. 4. In turbulence the phase is found at $\Phi \approx \pi / 2$ in the frequency range $f=$ $7-10 \mathrm{kHz}$. This is in good agreement with earlier observations using spatiotemporal fluctuation measurements [9]. In contrast, the phase shift in the controlled situation is much smaller with $\Phi \approx 0$, which is similar to the coherent drift mode case. Consequently, although the density fluctuation amplitude is not decreased the reduced phase shift leads to considerable reduction of the fluctuation driven cross-field transport to the coherent drift mode level. 


\section{SUMMARY AND DISCUSSION}

The mode-selective influence of drift wave dynamics was demonstrated using two control arrangements, which both drive parallel current patterns similar in shape and amplitude as the currents of coherent drift modes. Single coherent modes can be synchronized to the control signal over a frequency range around the intrinsic drift mode frequency. The width of the frequency range is found to be asymmetric and depends nonlinearly on the control amplitude when the driven current amplitude is larger than $50 \%$ of the drift wave current. The specific run of the curve is known as Arnold tongue and demonstrates the nonlinear character of interaction between the control and the drift wave. The synchronization acts as spatiotemporal control since it is not observed if the driven mode pattern or the direction of propagation is not the one of the drift mode. In drift wave turbulence the incoherent turbulent fluctuations are controlled and coherent fluctuations are observed, which show the characteristics of single coherent drift modes. Especially the phase between density and potential fluctuations decreases from $\pi / 2$, which results in maximum transport, to almost zero. Thus, the transport is strongly reduced to the level of transport caused by coherent drift modes.

[1] O. E. Garcia, J. Horacek, R. A. Pitts, et al., Plasma Phys. Controlled Fusion 48(1), L1 (2006).

[2] T. T. Ribeiro and B. Scott, Plasma Phys. Controlled Fusion 47(10), 1657 (2005).

[3] H. Schuster, ed., Handbook of Chaos Control (Wiley-VCH, 1999).

[4] A. K. Sen, J. S. Chiu, and P. Tham, Plasma Phys. Controlled Fusion 39, A333 (1997).

[5] T. Uckan, B. Richards, A. J. Wootton, et al., J. Nucl. Mater. 222, 663 (1995).

[6] C. Schröder, T. Klinger, D. Block, et al., Phys. Rev. Letters 86, 5711 (2001).

[7] C. Schröder, O. Grulke, T. Klinger, and V. Naulin, Phys. Plasmas 11(9), 4249 (2004).

[8] C. Schröder, O. Grulke, T. Klinger, and V. Naulin, Phys. Plasmas 12(4), 42103 (2005).

[9] T. Windisch, O. Grulke, and T. Klinger, Physica Scripta T122, 15 (2006). 
FIG. 1: Measurement of the parallel current density of a linear $m=2$ drift mode (left) and as driven by the control electrodes for the same mode number (right).

FIG. 2: Power spectral density of density fluctuations for a frequency scan of the control electrodes (top, left) and the control coil arrangement (top, right). The frequency of the control signal is indicated as the relative mismatch when compared to the drift mode frequency. Additionally the dependence of the control range on the control signal amplitude is shown for both cases (bottom).

FIG. 3: Power spectral density of plasma density fluctuations (left column), spatiotemporal evolution of density fluctuations as measured with an azimuthal 64 probe array (middle column), and frequencywavenumber spectra (right column). Top row: weakly developed turbulence. Bottom row: controlled turbulence.

FIG. 4: Phase spectrum between plasma density and plasma potential fluctuations for weakly developed turbulence (top) and controlled turbulence (bottom). The errorbars indicate the statistical confidence. 


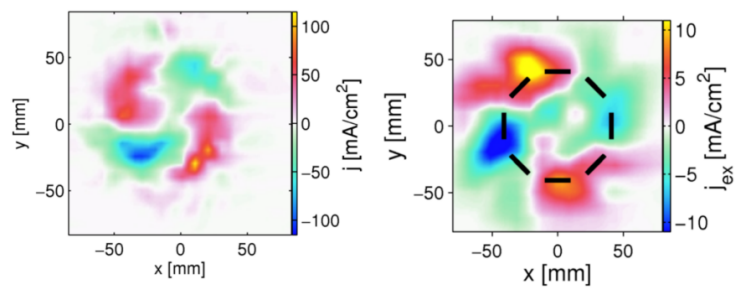

FIG. 1: width $=75 \mathrm{~mm}$
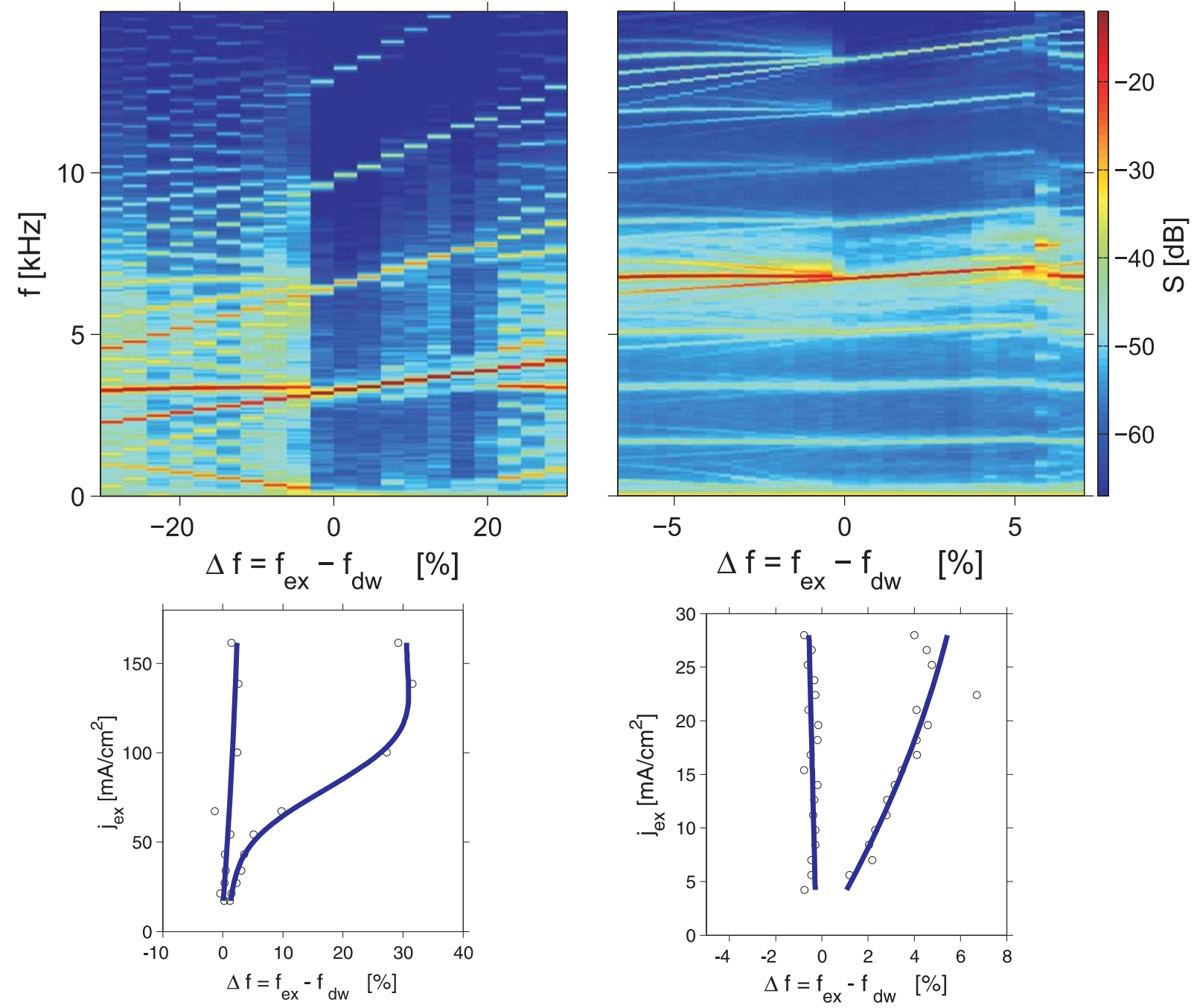

FIG. 2: width $=160 \mathrm{~mm}$ 

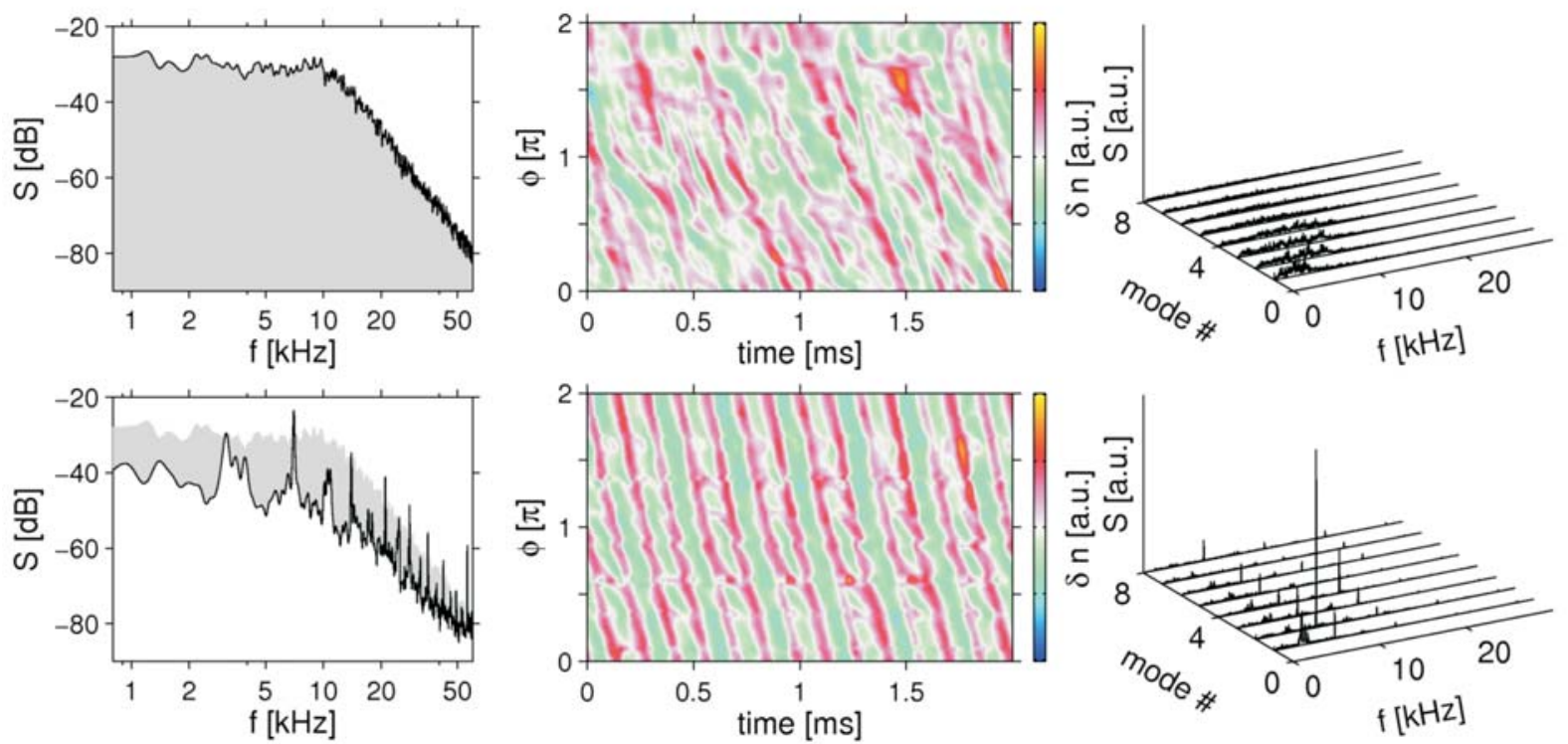

FIG. 3: width=160mm

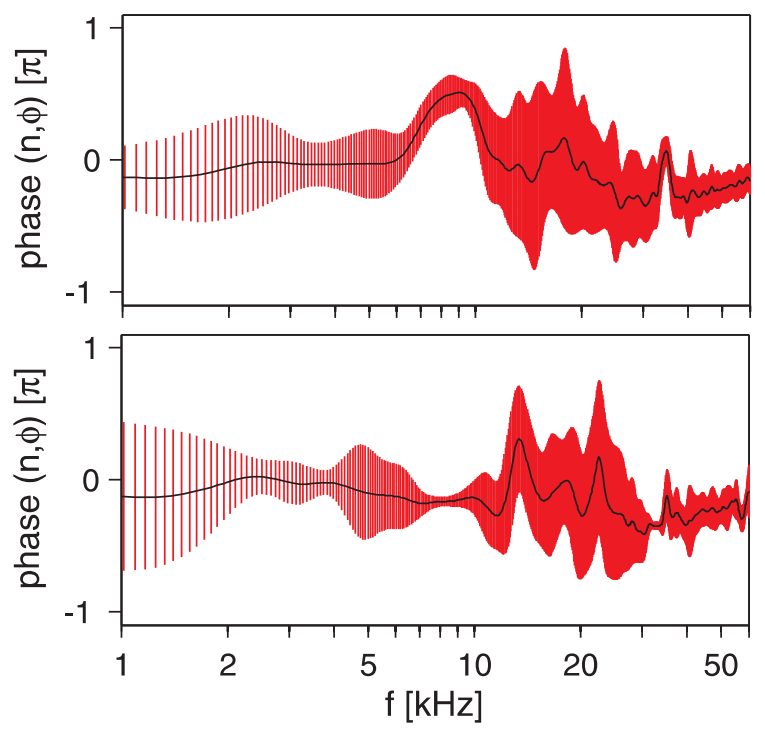

FIG. 4 : width $=75 \mathrm{~mm}$ 